\title{
A Note Concerning Equipotent Digraph Homomorphism Sets
}

\begin{abstract}
A. D. Parks ${ }^{1}$
${ }^{1} \hbar$ Laboratory, Electromagnetic and SensorSystems Department, 18444 Frontage Road Suite 327, Naval Surface Warfare Center Dahlgren Division, Dahlgren, VA 22448-5161

Abstract: Functor adjunctions are fundamental to category theory and have recently found applications in the empirical sciences. In this paper a functor adjunction on a special full subcategory of the category of digraphs is borrowed from mathematical biology and used to equate cardinalities of sets of homomorphisms between various types of digraphs and associated line digraphs. These equalities are especially useful for regular digraphs and are applied to obtain homomorphism set cardinality equalities for the classes of de Bruijn digraphs and Kautz digraphs. Such digraphs play important roles in bioinformatics and serve as architectures for distributed high performance computing networks.
\end{abstract}

Keywords: Category Theory, Functors, Adjunctions, Digraphs, Line Digraphs, Homomorphisms, De Bruijn Digraphs, Kautz Digraphs

\section{Introduction}

Every area of mathematics (e.g., group theory, topology) is described by numerous definitions, theorems, and constructions. However, many common mathematical concepts occur naturally with only slight variation in these various areas of mathematics. Category theory is that branch of mathematics which identifies and studies such common concepts and provides formal mechanisms for mapping them from one area of mathematics to another. More specifically, a category (e.g., the category of sets) consists of a class of objects (e.g., sets), morphisms between objects (e.g., maps between sets), an identity morphism for each object (e.g., the set identity map), and a rule for associatively composing morphisms (e.g., composition of maps). Functors provide formal maps between categories (e.g., from the category of groups to the category of sets) by associating objects and morphisms in different categories subject to the constraints that morphism composition and object identities are preserved.

Because of its generality, category theory has found application in recent years in such diverse areas as physics (e.g., [1-4]), design specification (e.g., [5,6]), data fusion (e.g., [7]), computer science (e.g., [8]), computer security (e.g., $[9,10])$, systems engineering (e.g., [11]), manufacturing (e.g., [12]), theoretical biology (e.g., [13]), and artificial intelligence (e.g., [14]). Of special interest here is the recent application of category theory by Haruna and Gunji (HG) to theoretical biology $[15,16]$. There two functors are defined on a full subcategory of the category of digraphs and are shown to form an adjoint pair (i.e., a functor adjunction) - an important category theoretic concept analogous to that of mutually inverse functions.

Although this adjoint pair of functors has important consequences for biological systems, the objective of this paper is to exploit HG's functor adjunction for the purpose of establishing equalities between the cardinalities of homomorphism sets for various types of digraphs and associated line digraphs. These equalities are especially useful for regular digraphs and they are applied to obtain results for the classes of de Bruijn digraphs and Kautz digraphs. Both de Bruijn and Kautz digraphs play important roles in bioinformatics (e.g., [17]) and serve as efficient architectures for distributed high performance computing networks (e.g., [18]).

To make this paper relatively self-contained, basic graph and category theoretic definitions that are used in this paper are summarized in the next section and results from HG's research that are relevant to this work are presented in section 3 . The theory developed in section 3 is used in section 4 to establish this paper's main theorems concerning equalities between 
equipotent digraph homomorphism sets. These main results are applied to dicycles, de Bruijn digraphs, and Kautz digraphs in section 5. Closing remarks comprise the final section of this paper.

\section{Basic Definitions}

A digraph (or directed graph) $D$ is a 4-tuple $D=$ $\left(A, N, \partial_{0}, \partial_{1}\right)$, where $A$ is a set of arcs, $N$ is a set of nodes, $\partial_{0}$ is a map that sends each arc to its source node, and $\partial_{1}$ is a map that sends each arc to its target node. A source (target) node is adjacent to (from) a target (source) node and the in-degree (out-degree) $\delta^{+}(v)\left(\delta^{-}(v)\right)$ of a node $v$ is the number nodes adjacent to (from) $v$. $D$ is $k$-regular if $\delta^{+}(v)=k=$ $\delta^{-}(v)$ for each of its nodes. If $\theta$ is a partition of the node set $N$ of $D$ into $n$ non-empty subsets $N_{j}, j \in J \equiv$ $\{1,2, \cdots, n\}$, then $D / \theta$ is the associated quotient digraph of $D$ with node set $J$ and arc set $\left\{\left(j, j^{\prime}\right)\right.$ : when $(u, v) \in A$ and $\left.u \in N_{j}, v \in N_{j^{\prime}}\right\}$. The line digraph of digraph $D=\left(A, N, \partial_{0}, \partial_{1}\right)$ is the digraph which has $A$ as its node set and arc set $\{(x, y) \in A \times$ $\left.A: \partial_{1} x=\partial_{0} y\right\}$.

A digraph homomorphism from $D=\left(A, N, \partial_{0}, \partial_{1}\right)$ to $D^{\prime}=\left(A^{\prime}, N^{\prime}, \partial_{0}^{\prime}, \partial_{1}^{\prime}\right)$ is a pair of maps $\left(\varphi_{A}, \varphi_{N}\right)$, where $\varphi_{A}: A \rightarrow A^{\prime}$ and $\varphi_{N}: N \rightarrow N^{\prime}$, such that $\varphi_{N} \partial_{i}=$ $\partial_{i}^{\prime} \varphi_{A}, i \in\{0,1\}$. The number of injective digraph homomorphisms of $D$ into $K$ is $\operatorname{inj}(D, K)$ and an endomorphism on $D$ is a digraph homomorphism from $D$ to itself. $\operatorname{End}(D)$ is the number of $D$ endomorphisms and $D$ is said to be rigid when $\operatorname{End}(D)=1$.

A category $\boldsymbol{C}$ consists of a collection $O b j_{C}$ of objects such that:

(1) for every pair of objects $X, Y$ in $O b j_{C}$ there is a (possibly empty) set $\operatorname{Mor}_{C}(X, Y)$ of morphisms from $X$ to $Y$;

(2) for any $X, Y, Z$ in $O b j_{C}$ there is a composition of morphisms

$\circ: \operatorname{Mor}_{C}(X, Y) \times \operatorname{Mor}_{C}(Y, Z) \rightarrow \operatorname{Mor}_{C}(X, Z)$ given by $(f, g) \mapsto g \circ f$ with the properties:

a. for every $X$ in $O b j_{C}$ there is an identity morphism $1_{X} \in \operatorname{Mor}_{C}(X, X)$ such that for

$$
\begin{array}{lrr}
\quad f \in \operatorname{Mor}_{C}(X, Y) & \text { and } & g \in \\
\operatorname{Mor}_{C}(Y, X), \quad f \circ 1_{X}= & f & \text { and } \\
1_{X} \circ g=g ; \text { and } & &
\end{array}
$$

b. when defined, composition of morphisms is associative, i.e., $(f \circ g) \circ h=f \circ(g \circ h)$.

A morphism $f: X \rightarrow Y$ is an isomorphism if there exists a morphism $g: Y \rightarrow X$ such that $f \circ g=1_{Y}$ and $g \circ f=1_{X}$. A category $\boldsymbol{C}$ is a full subcategory of category $\boldsymbol{D}$ if every object of $\boldsymbol{C}$ is an object of $\boldsymbol{D}$, $\operatorname{Mor}_{C}(X, Y)=\operatorname{Mor}_{D}(X, Y)$ for all objects $X$ and $Y$ in $\boldsymbol{C}$, for every object $X$ in $\boldsymbol{C}$ the identity morphism $1_{X}$ is the same in $\boldsymbol{D}$ as it is in $\boldsymbol{C}$, and the composite of two morphisms in $\boldsymbol{C}$ is the same as their composite in $\boldsymbol{D}$.

Examples of categories are the category Set (where $O b j_{S e t}$ is the collection of all sets, the morphisms are the ordinary mappings between sets, and $\circ$ is the usual composition of maps), the category Grp (where $O b j_{G r p}$ is the collection of all groups, the morphisms are the ordinary group homomorphisms, and $\circ$ is the usual composition of group homomorphisms), and the category $\boldsymbol{D} \boldsymbol{g} \boldsymbol{p}$ of digraphs (where $O b j_{\boldsymbol{D g} \boldsymbol{p}}$ is the collection of all digraphs, the morphisms are digraph homomorphisms, and $\circ$ is composition of digraph homomorphisms). It is easily verified that $\boldsymbol{S e t}, \boldsymbol{G r p}$, and $\boldsymbol{D} \boldsymbol{g} \boldsymbol{p}$ satisfy items (1), (2a), and (2b) above.

Functors can be regarded as morphisms between categories and - in a sense - they provide a "picture" of what one category looks like inside another. If $F$ is a covariant functor - or simply a functor (contravariant functors are not used here) - from category $\boldsymbol{C}$ to category $\boldsymbol{D}$ (denoted $F: \boldsymbol{C} \rightarrow \boldsymbol{D}$ ), then it assigns to every $X$ in $O b j_{C}$ an object $F X$ in $O b j_{D}$ and to every $f \in \operatorname{Mor}_{C}(X, Y)$ an $F f \in \operatorname{Mor}_{D}(F X, F Y)$ such that:

(3) $F 1_{X}=1_{F X}$ for every $X$ in $O b j_{C}$; and

(4) when $f \circ g=h$ is defined in $C$, then $F f \circ F g=$ $F h$ is defined in $\boldsymbol{D}$ and $F f \circ F g=F(f \circ g)=$ $F h$.

If $F$ is a functor such that $F: \boldsymbol{C} \rightarrow \boldsymbol{C}$, then $F$ is an endofunctor.

Simple examples of functors are the identity functor $1_{\boldsymbol{C}}: \boldsymbol{C} \rightarrow \boldsymbol{C}$ (which makes the assignments $1_{\boldsymbol{C}} X=X$ for every $X$ in $O b j_{C}$ and $1_{c} f=f$ for every $f \in$ $\left.\operatorname{Mor}_{C}(X, Y)\right)$ and the forgetful functor $U: \boldsymbol{G r p} \rightarrow \boldsymbol{S e t}$ (which assigns to every group $G$ in $O b j_{G r p}$ its underlying set $U G$ in $O b j_{\text {Set }}$ and to each homomorphism $f \in \operatorname{Mor}_{G r p}(G, H)$ the set map $U f \in \operatorname{Mor}_{\text {Set }}(U G, U H)$ - i.e., $U$ forgets the group structure going from Grp to Set). It can be determined by inspection that these functors satisfy the required properties given above by items (3) and (4). It is also straightforward to see that if $F: \boldsymbol{C} \rightarrow \boldsymbol{D}$ and $G: \boldsymbol{D} \rightarrow \boldsymbol{E}$ are functors, then their composition $G F: \boldsymbol{C} \rightarrow \boldsymbol{E}$ is also a functor. 
Let $F, G: \boldsymbol{C} \rightarrow \boldsymbol{D}$ be functors. A natural transformation from $F$ to $G$ is a morphism $\varphi_{X}: F X \rightarrow$ $G X$ in $\boldsymbol{D}$ for every $X$ in $\boldsymbol{C}$ such that if $f \in \operatorname{Mor}_{\boldsymbol{C}}(X, Y)$, then $\varphi_{Y} \circ F f=G f \circ \varphi_{X}$. If $\varphi_{X}$ is an isomorphism, then $\varphi_{X}$ is a natural isomorphism. An adjunction between functors $F: \boldsymbol{D} \rightarrow \boldsymbol{C}$ and $G: \boldsymbol{C} \rightarrow \boldsymbol{D}$, denoted by $F \dashv G$, is a family of natural isomorphisms $\Phi_{X, Y}: \operatorname{Mor}_{C}(F X, Y) \rightarrow \operatorname{Mor}_{D}(X, G Y)$ for all objects $X$ in $\boldsymbol{D}$ and all objects $Y$ in $\boldsymbol{C}$, denoted by $\operatorname{Mor}_{C}(F X, Y) \cong \operatorname{Mor}_{D}(X, G Y)$.

\section{The Endofunctors $\mathcal{L}$ and $\mathcal{R}$ on Category $\mathcal{H}$ and Their Adjunction}

The results found in HG's research that are required to provide the main results of this paper are presented in this section. However, it should be noted that HG omitted in $[15,16]$ explicit proofs that their "transformation operators" $\mathcal{L}$ and $\mathcal{R}$ are endofunctors. For the sake of completeness, the proofs that $\mathcal{L}$ and $\mathcal{R}$ are endofunctors - along with appropriate homomorphism definitions - are developed in this section. Also note that for the purpose of mnemonics, HG's $\mathcal{R}(\mathcal{L})$ is the $\mathcal{L}(\mathcal{R})$ used herein.

Fundamental to this note is the category $\mathcal{H}$ defined by $\mathrm{HG}$ which has as its objects digraphs $D=$ $\left(A, N, \partial_{0}, \partial_{1}\right)$ with the property that for every $v \in N$ there exists $x, y \in A$ such that $\partial_{1} x=v=\partial_{0} y$ and has as its morphisms the associated digraph homomorphisms. The following lemma follows trivially from the definition of full subcategory and is stated without proof.

Lemma 1. $\mathcal{H}$ is a full subcategory of $\mathbf{D g p}$.

Let $\mathcal{L}$ transform digraph $D=\left(A, N, \partial_{0}, \partial_{1}\right)$ into another digraph $\mathcal{L} D=\left(\mathcal{L} A, \mathcal{L} N, \partial_{0}^{\mathcal{L}}, \partial_{1}^{\mathcal{L}}\right)$ according to $\mathcal{L} A=\left\{(x, y) \in A \times A: \partial_{1} x=\partial_{0} y\right\}, \quad \mathcal{L} N=A, \quad$ with $\partial_{0}^{\mathcal{L}}(x, y)=x$ and $\partial_{1}^{\mathcal{L}}(x, y)=y$ for $(x, y) \in \mathcal{L} A$.

Lemma 2. $\mathcal{L} D$ is the line digraph of $D$.

Proof of Lemma 2. This assertion follows directly from the definitions of $\mathcal{L} D$ and line digraph.

If $D^{\prime}=\left(A^{\prime}, N^{\prime}, \partial_{0}^{\prime}, \partial_{1}^{\prime}\right)$, then let $\theta \equiv\left(\varphi_{A}, \varphi_{N}\right)$ be a homomorphism from $D$ to $D^{\prime}$ such that $\varphi_{A}: A \rightarrow A^{\prime}$ and $\varphi_{N}: N \rightarrow N^{\prime}$ and define $\mathcal{L} \theta=\left(\mathcal{L} \varphi_{A}, \mathcal{L} \varphi_{N}\right)$, where $\mathcal{L} \varphi_{A}: \mathcal{L} A \rightarrow \mathcal{L} A^{\prime} \quad$ according $(x, y) \stackrel{\mathcal{L} \varphi_{A}}{\longrightarrow}\left(\varphi_{A}(x), \varphi_{A}(y)\right)$ and $\mathcal{L} \varphi_{N}=\varphi_{A}$.

Lemma 3. $\mathcal{L} \theta$ is a digraph homomorphism.

Proof of Lemma 3. If $\mathcal{L} \theta$ is a homomorphism from $\mathcal{L} D$ to $\mathcal{L} D^{\prime}$, then $\mathcal{L} \varphi_{N} \partial_{i}^{\mathcal{L}}=\partial_{i}^{\prime \mathcal{L}} \mathcal{L} \varphi_{A}, i \in\{0,1\}$. "Diagram chasing" shows that this is indeed the case: for $i=0,(x, y) \stackrel{\mathcal{L} \varphi_{A}}{\longrightarrow}\left(\varphi_{A}(x), \varphi_{A}(y)\right) \stackrel{\partial_{0}^{\prime} \mathcal{L}}{\longrightarrow} \varphi_{A}(x) \stackrel{\mathcal{L} \varphi_{N}}{\longleftarrow} x$
$\stackrel{\partial_{0}^{\mathcal{L}}}{\leftarrow}(x, y)$ and similarly for $i=1$.

Lemma 4. $\mathcal{L}$ is an endofunctor on $\mathcal{H}$.

Proof of Lemma 4. That $\mathcal{L} D$ is an object in $\mathcal{H}$ is shown to be true by Proposition 5.7 (iv) in [15]. To show that item (3) in section 2 is true for $\mathcal{L}$, let $D=D^{\prime}$ so that $\varphi_{A}$ and $\varphi_{N}$ are the identity maps $i d_{A}$ and $i d_{N}$, respectively, in which case $\theta \equiv\left(i d_{A}, i d_{N}\right)=$ $1_{D}$. Then $\mathcal{L} \theta=\mathcal{L} 1_{D}=\left(\mathcal{L i d}_{A},{\mathcal{L} i d_{N}}\right)$. Since $\mathcal{L i d}_{A}$ and $\mathcal{L i d}_{N}$ map according to

$$
(x, y) \stackrel{\mathcal{L i d}_{A}}{\longrightarrow}\left(i d_{A}(x), i d_{A}(y)\right)=(x, y)
$$

and

$$
x \stackrel{\mathcal{L i d}_{N}}{\longrightarrow} i d_{A}(x)=x
$$

then

$\left(\mathcal{L i d}_{A}, \mathcal{L} i d_{N}\right)$ is the identity morphism $1_{\mathcal{L} D}$ on $\mathcal{L} D$. It follows that $\mathcal{L} 1_{D}=1_{\mathcal{L D}}$. To show that item (4) in section 2 is true for $\mathcal{L}$, let $\theta: D \rightarrow D^{\prime}$ and $\theta^{\prime}: D^{\prime} \rightarrow D^{\prime \prime}$ be digraph homomorphisms. Then - since $\varphi_{A}$ and $\varphi_{N}$ compose as maps - the composition

$$
\theta^{\prime} \theta=\left(\varphi_{A}^{\prime} \varphi_{A}, \varphi_{N}^{\prime} \varphi_{N}\right)
$$

implies

$$
\varphi_{N}^{\prime \prime} \partial_{i}=\partial_{i}^{\prime \prime} \varphi_{A}^{\prime \prime}, i \in\{0,1\},
$$

where $\varphi_{N}^{\prime \prime}=\varphi_{N}^{\prime} \varphi_{N}$ and $\varphi_{A}^{\prime \prime}=\varphi_{A}^{\prime} \varphi_{A}$. Consequently, $\theta^{\prime} \theta$ is defined in $\mathcal{H}$. Consider the composition $\mathcal{L} \theta^{\prime} \mathcal{L} \theta$. "Diagram chasing" reveals that

$$
\begin{aligned}
(x, y) \stackrel{\mathcal{L} \varphi_{A}}{\longrightarrow}\left(\varphi_{A}(x), \varphi_{A}(y)\right) \stackrel{\mathcal{L} \varphi_{A}^{\prime}}{\longrightarrow}\left(\varphi_{A}^{\prime} \varphi_{A}(x), \varphi_{A}^{\prime} \varphi_{A}(y)\right) \\
\\
\stackrel{\partial_{0}^{\prime \prime}}{\longrightarrow} \varphi_{A}^{\prime} \varphi_{A}(x) \stackrel{\mathcal{L} \varphi_{N}^{\prime}}{\longleftarrow} \varphi_{A}(x) \stackrel{\mathcal{L} \varphi_{N}}{\longleftarrow} x \\
\stackrel{\partial_{0}^{\mathcal{L}}}{\leftarrow}(x, y)
\end{aligned}
$$

and similarly for $i=1$. Thus, $\mathcal{L} \varphi_{N}^{\prime} \mathcal{L} \varphi_{N} \partial_{i}^{\mathcal{L}}=$ $\partial_{i}^{\prime \prime} \mathcal{L} \mathcal{L} \varphi_{A}^{\prime} \mathcal{L} \varphi_{A}, i \in\{0,1\}$, which shows that $\mathcal{L} \theta^{\prime} \mathcal{L} \theta$ is also defined in $\mathcal{H}$. Now observe that since

$$
\begin{aligned}
(x, y) \stackrel{\mathcal{L} \varphi_{A}}{\longrightarrow}\left(\varphi_{A}(x), \varphi_{A}(y)\right) \stackrel{\mathcal{L} \varphi_{A}^{\prime}}{\longrightarrow}\left(\varphi_{A}^{\prime} \varphi_{A}(x), \varphi_{A}^{\prime} \varphi_{A}(y)\right) \\
\stackrel{\mathcal{L} \varphi_{A}^{\prime} \varphi_{A}}{\longleftarrow}(x, y),
\end{aligned}
$$

then $\mathcal{L} \varphi_{A}^{\prime} \mathcal{L} \varphi_{A}=\mathcal{L} \varphi_{A}^{\prime} \varphi_{A}$. Also, since $\mathcal{L} \varphi_{N}^{\prime} \mathcal{L} \varphi_{N}(x)=$ $\varphi_{A}^{\prime} \varphi_{A}(x)=\mathcal{L} \varphi_{N}^{\prime} \varphi_{N}$, then $\mathcal{L} \theta^{\prime} \mathcal{L} \theta=\left(\mathcal{L} \varphi_{A}^{\prime} \mathcal{L} \varphi_{A}, \mathcal{L} \varphi_{N}^{\prime} \mathcal{L} \varphi_{N}\right)=$ $\left(\mathcal{L} \varphi_{A}^{\prime} \varphi_{A}, \mathcal{L} \varphi_{N}^{\prime} \varphi_{N}\right)=\mathcal{L} \theta^{\prime} \theta$.

For obvious reasons, $\mathcal{L}$ is called here the line graph functor on $\mathcal{H}$.

Now let $\mathcal{R}$ transform $D$ into another digraph $\mathcal{R} D=\left(\mathcal{R} A, \mathcal{R} N, \partial_{0}^{\mathcal{R}}, \partial_{1}^{\mathcal{R}}\right), \quad$ where $\quad \mathcal{R} A=N, \mathcal{R} N=$ 
$T / \sim, T \equiv\left\{(u, v) \in N \times N: \exists x \in A, \partial_{0} x=u, \partial_{1} x=\right.$ $v\}$, and $\sim$ is the equivalence relation generated by the relation $(u, v) \rho(z, w)$ if and only if $u=z$ or $v=w$. Denote an $\sim$ equivalence class by $[(u, v)]_{\sim}$, where $(u, v)$ is a representative element in the equivalence class, and define the source and target maps for $u \in \mathcal{R} A \quad$ as $\quad \partial_{0}^{\mathcal{R}} u=\left[\left(\partial_{0} x, \partial_{1} x\right)\right]_{\sim} \quad$ and $\quad \partial_{1}^{\mathcal{R}} u=$ $\left[\left(\partial_{0} y, \partial_{1} y\right)\right]_{\sim}$, where $\partial_{1} x=u=\partial_{0} y$ for $x, y \in A$. As before let $\theta \equiv\left(\varphi_{A}, \varphi_{N}\right)$ be a homomorphism from $D$ to $D^{\prime}$ and define $\mathcal{R} \theta=\left(\mathcal{R} \varphi_{A}, \mathcal{R} \varphi_{N}\right)$, where $\mathcal{R} \varphi_{A}=$ $\varphi_{N}$ and $\mathcal{R} \varphi_{N}: \mathcal{R} N \rightarrow \mathcal{R} N^{\prime}$ according to

$$
\left[\left(\partial_{0} x, \partial_{1} x\right)\right]_{\sim}=\left[\left(\partial_{0} x, u\right)\right]_{\sim} \stackrel{\mathcal{R} \varphi_{N}}{\longrightarrow}\left[\left(\partial_{0}^{\prime} x^{\prime}, \varphi_{N}(u)\right)\right]_{\sim}
$$

and

$$
\left[\left(\partial_{0} y, \partial_{1} y\right)\right]_{\sim}=\left[\left(u, \partial_{1} y\right)\right]_{\sim} \stackrel{\mathcal{R} \varphi_{N}}{\longrightarrow}\left[\left(\varphi_{N}(u), \partial_{1}^{\prime} y^{\prime}\right)\right]_{\sim} .
$$

Lemma 5. $\mathcal{R} \theta$ is a digraph homomorphism.

Proof of Lemma 5. If $\mathcal{R} \theta$ is a homomorphism from $\mathcal{R} D$ to $\mathcal{R} D^{\prime}$, then $\mathcal{R} \varphi_{N} \partial_{i}^{\mathcal{R}}=\partial_{i}^{\mathcal{R}} \mathcal{R} \varphi_{A}, i \in\{0,1\}$. "Diagram chasing" shows that this is the case: for $i=0, \quad u \stackrel{\partial_{0}^{\mathcal{R}}}{\longrightarrow}\left[\left(\partial_{0} x, u\right)\right]_{\sim} \stackrel{\mathcal{R} \varphi_{N}}{\longrightarrow}\left[\left(\partial_{0}^{\prime} x^{\prime}, \varphi_{N}(u)\right)\right]_{\sim}$ $\stackrel{\partial_{0}^{\prime} \mathcal{R}}{\longleftarrow} \varphi_{N}(u) \stackrel{\mathcal{R} \varphi_{A}}{\longleftarrow} u$ and for $i=1, \quad u \stackrel{\partial^{\mathcal{R}}}{\rightarrow}\left[\left(u, \partial_{1} y\right)\right]_{\sim}$ $\stackrel{\mathcal{R} \varphi_{N}}{\longrightarrow}\left[\left(\varphi_{N}(u), \partial_{1}^{\prime} y^{\prime}\right)\right]_{\sim} \stackrel{\partial_{1}^{\mathcal{R}^{\prime}}}{\longleftarrow} \varphi_{N}(u) \stackrel{\mathcal{R} \varphi_{A}}{\longleftarrow} u$.

Lemma 6. $\mathcal{R}$ is an endofunctor on $\mathcal{H}$.

Proof of Lemma 6. That $\mathcal{R D}$ is an object in $\mathcal{H}$ when $D$ is an object in $\mathcal{H}$ is shown to be true by Proposition 5.7 (iii) in [15]. To show that item (3) in section 2 is true for $\mathcal{R}$, let $D=D^{\prime}$ so that $\varphi_{A}$ and $\varphi_{N}$ are the identity maps $i d_{A}$ and $i d_{N}$, respectively, in which case $\theta \equiv\left(i d_{A}, i d_{N}\right)=1_{D} . \quad$ Then $\quad \mathcal{R} \theta=\mathcal{R} 1_{D}=$ $\left(\mathcal{R i d}_{A}, \mathcal{R i d}_{N}\right)$. Since $\mathcal{R i d}_{A}$ maps as $u \stackrel{\mathcal{R} \varphi_{A}}{\longrightarrow} i d_{N}(u)=$ $u$ and $\mathcal{R} i d_{N}$ maps according to

$$
\left[\left(\partial_{0} x, u\right)\right]_{\sim} \stackrel{\mathcal{R} \varphi_{N}}{\longrightarrow}\left[\left(\partial_{0} x, i d_{N}(u)\right)\right]_{\sim}=\left[\left(\partial_{0} x, u\right)\right]_{\sim}
$$

and

$$
\left[\left(u, \partial_{1} y\right)\right]_{\sim} \stackrel{\mathcal{R} \varphi_{N}}{\longrightarrow}\left[\left(i d_{N}(u), \partial_{1} y\right)\right]_{\sim}=\left[\left(u, \partial_{1} y\right)\right]_{\sim},
$$

then $\left(\mathcal{R} i d_{A}, \mathcal{R} i d_{N}\right)$ is the identity morphism $1_{\mathcal{R} D}$ on $\mathcal{R} D$. Thus, $\mathcal{R} 1_{D}=1_{\mathcal{R} D}$. To show that item (4) in section 2 is true for $\mathcal{R}$, let $\theta: D \rightarrow D^{\prime}$ and $\theta^{\prime}: D^{\prime} \rightarrow D^{\prime \prime}$ be digraph homomorphisms. Then - since $\varphi_{A}$ and $\varphi_{N}$ compose as maps - the composition $\theta^{\prime} \theta=$ $\left(\varphi_{A}^{\prime} \varphi_{A}, \varphi_{N}^{\prime} \varphi_{N}\right) \quad$ implies $\varphi_{N}^{\prime \prime} \partial_{i}=\partial_{i}^{\prime \prime} \varphi_{A}^{\prime \prime}, i \in\{0,1\}$, where $\varphi_{N}^{\prime \prime}=\varphi_{N}^{\prime} \varphi_{N}$ and $\varphi_{A}^{\prime \prime}=\varphi_{A}^{\prime} \varphi_{A}$. Consequently, $\theta^{\prime} \theta$ is defined in $\mathcal{H}$. Consider the composition $\mathcal{R} \theta^{\prime} \mathcal{R} \theta$. "Diagram chasing" reveals that

$$
\begin{aligned}
& u \stackrel{\mathcal{R} \varphi_{A}}{\longrightarrow} \varphi_{N}(u) \stackrel{\mathcal{R} \varphi_{A}^{\prime}}{\longrightarrow} \varphi_{N}^{\prime} \varphi_{N}(u) \\
& \stackrel{\partial_{0}^{\prime \prime}}{\longrightarrow}\left[\left(\partial_{0}^{\prime \prime} x^{\prime \prime}, \varphi_{N}^{\prime} \varphi_{N}(u)\right)\right]_{\sim} \stackrel{\mathcal{R} \varphi_{N}^{\prime}}{\longleftarrow}\left[\left(\partial_{0}^{\prime} x^{\prime}, \varphi_{N}(u)\right)\right]_{\sim} \\
& \stackrel{\mathcal{R} \varphi_{N}}{\longleftarrow}\left[\left(\partial_{0} x, u\right)\right]_{\sim} \stackrel{\partial_{0}^{\mathcal{R}}}{\leftarrow} u
\end{aligned}
$$

and similarly for $i=1$. Thus, $\mathcal{R} \varphi_{N}^{\prime} \mathcal{R} \varphi_{N} \partial_{i}^{\mathcal{R}}=$ $\partial_{i}^{\prime \prime} \mathcal{R} \mathcal{R} \varphi_{A}{ }^{\prime} \mathcal{R} \varphi_{A}, i \in\{0,1\}$, which shows that $\mathcal{R} \theta^{\prime} \mathcal{R} \theta$ is also defined in $\mathcal{H}$. Now observe that since

$$
u \stackrel{\mathcal{R} \varphi_{A}}{\longrightarrow} \varphi_{N}(u) \stackrel{\mathcal{R} \varphi_{A}^{\prime}}{\longrightarrow} \varphi_{N}^{\prime} \varphi_{N}(u) \stackrel{\mathcal{R} \varphi_{A}^{\prime} \varphi_{A}}{\longleftrightarrow} u,
$$

then $\mathcal{R} \varphi_{A}{ }^{\prime} \mathcal{R} \varphi_{A}=\mathcal{R} \varphi_{A}^{\prime} \varphi_{A}$. Also, since

$$
\begin{gathered}
{\left[\left(\partial_{0} x, u\right)\right]_{\sim} \stackrel{\mathcal{R} \varphi_{N}^{\prime} \mathcal{R} \varphi_{N}}{\longrightarrow}\left[\left(\partial_{0}^{\prime \prime} x^{\prime \prime}, \varphi_{N}^{\prime} \varphi_{N}(u)\right)\right]_{\sim}} \\
\stackrel{\mathcal{R} \varphi_{N}^{\prime} \varphi_{N}}{\longleftarrow}\left[\left(\partial_{0} x, u\right)\right]_{\sim},
\end{gathered}
$$

then

$\mathcal{R} \theta^{\prime} \mathcal{R} \theta=\left(\mathcal{R} \varphi_{A}^{\prime} \mathcal{R} \varphi_{A}, \mathcal{R} \varphi_{N}^{\prime} \mathcal{R} \varphi_{N}\right)=$

$\left(\mathcal{R} \varphi_{A}^{\prime} \varphi_{A}, \mathcal{R} \varphi_{N}^{\prime} \varphi_{N}\right)=\mathcal{R} \theta^{\prime} \theta$

Lemma 7. $\mathcal{R} \mathcal{L} \cong 1_{\mathcal{H}}$.

Proof of Lemma 7. This is shown to be true in Theorem 5.9 in [15].

$\mathcal{R}$ is called here the digraph return functor on $\mathcal{H}$ since - from Lemma $7-\mathcal{R} \mathcal{L} D \cong D$, i.e. the action of $\mathcal{R}$ upon the line graph $\mathcal{L} D$ of $D$ "returns" $D$ to an isomorphic copy $D^{\prime}$ of itself in $\mathcal{H}$. Denote this isomorphism by $D \approx D^{\prime}$.

Lemma 8. $\mathcal{R} \dashv \mathcal{L}$, that is $\operatorname{Mor}_{\mathcal{H}}(\mathcal{R} D, K) \cong$ $\operatorname{Mor}_{\mathcal{H}}(D, \mathcal{L} K)$ for all objects $D$ and $K$ in $\mathcal{H}$.

Proof of Lemma 8. This is a restatement of Theorem 5.8 in [15].

\section{Main Results}

The above lemmas lead to the following main results which equate the cardinalities of homomorphism sets between digraphs, line digraphs, and associated quotient digraphs.

Theorem 9. For any digraphs $D$ and $K$ in $\mathcal{H}$, $\left|\operatorname{Mor}_{\mathcal{H}}(\mathcal{R} D, K)\right|=\left|\operatorname{Mor}_{\mathcal{H}}(D, \mathcal{L} K)\right|$.

Proof of Theorem 9. This result follows directly from Lemma 8 because the associated families of natural isomorphisms $\Phi_{D, K}: \operatorname{Mor}_{\mathcal{H}}(\mathcal{R} D, K) \rightarrow$ $\operatorname{Mor}_{\mathcal{H}}(D, \mathcal{L} K)$ are bijections between the morphism sets $\operatorname{Mor}_{\mathcal{H}}(\mathcal{R D}, K)$ and $\operatorname{Mor}_{\mathcal{H}}(D, \mathcal{L} K)$. Consequently, these morphism sets have the same cardinality.

Theorem 10. For any objects $D$ and $K$ in $\mathcal{H}$,

$$
\sum_{\theta \in \Pi_{\mathcal{R} D}} \operatorname{inj}(\mathcal{R} D / \theta, K)=\sum_{\varphi \in \Pi_{D}} i n j(D / \varphi, \mathcal{L} K),
$$


where $\Pi_{\mathcal{R} D}$ and $\Pi_{D}$ are the sets of all partitions of the $\mathcal{R} D$ and $D$ node sets, respectively.

Proof of Theorem 10. Application of Corollary 1.27 in [19] to Theorem 9 yields $\sum_{\theta \in \Pi_{\mathcal{R} D}} \operatorname{inj}(\mathcal{R} D / \theta, K)=$ $\left|\operatorname{Mor}_{\mathcal{H}}(\mathcal{R} D, K)\right|=\left|\operatorname{Mor}_{\mathcal{H}}(D, \mathcal{L} K)\right|=$

$\sum_{\varphi \in \Pi_{D}} \operatorname{inj}(D / \varphi, \mathcal{L} K)$, from which the desired result follows.

Corollary 11. For any object $K$ in $\mathcal{H}$,

$$
\sum_{\theta \in \Pi_{K}} \operatorname{inj}(K / \theta, K)=\sum_{\varphi \in \Pi_{\mathcal{L} K}} \operatorname{inj}(\mathcal{L} K / \varphi, \mathcal{L} K),
$$

where $\Pi_{K}$ and $\Pi_{\mathcal{L} K}$ are the sets of all partitions of the $K$ and $\mathcal{L} K$ node sets, respectively.

Proof of Corollary 11. Let $D=\mathcal{L} K$ in Theorem 10 to obtain

$\sum_{\theta \in \Pi_{\mathcal{R} \mathcal{L} K}} \operatorname{inj}(\mathcal{R} \mathcal{L} K / \theta, K)=\sum_{\varphi \in \Pi_{\mathcal{L} K}} \operatorname{inj}(\mathcal{L} K / \varphi, \mathcal{L} K)$.

But from Lemma $7, \mathcal{R} \mathcal{L} K=K^{\prime}$ so that this now becomes

$\sum_{\theta \in \Pi_{K}} \operatorname{inj}\left(K^{\prime} / \theta, K\right)=\sum_{\varphi \in \Pi_{\mathcal{L K}}} \operatorname{inj}(\mathcal{L} K / \varphi, \mathcal{L} K)$.

Since $K \approx K^{\prime}$, then $K^{\prime} / \theta \approx K / \theta$. The result follows from the fact that there is a one-to-one correspondence between the set of injective homomorphisms from $K^{\prime} / \theta$ into $K$ and the set of injective homomorphisms from $K / \theta$ into $K$.

Theorem 12. If $K$ is an object in $\mathcal{H}$, then $\operatorname{End}(K)=$ $\operatorname{End}(\mathcal{L} K)$.

Proof of Theorem 12. Let $D=\mathcal{L} K$ in Theorem 9 in which case $\left|\operatorname{Mor}_{\mathcal{H}}(\mathcal{R} \mathcal{L} K, K)\right|=\left|\operatorname{Mor}_{\mathcal{H}}(\mathcal{L} K, \mathcal{L} K)\right| \equiv$ $\operatorname{End}(\mathcal{L} K)$. But from Lemma 7, $\mathcal{R} \mathcal{L} K=K^{\prime}$ so that now $\quad\left|\operatorname{Mor}_{\mathcal{H}}(\mathcal{R L} K, K)\right|=\left|\operatorname{Mor}_{\mathcal{H}}\left(K^{\prime}, K\right)\right|=$ $\operatorname{End}(\mathcal{L} K)$. Because $K \approx K^{\prime}$ there is a one-to-one correspondence between the morphisms in set $\operatorname{Mor}_{\mathcal{H}}\left(K^{\prime}, K\right)$ and those in $\operatorname{Mor}_{\mathcal{H}}(K, K)$ Consequently, $\left|\operatorname{Mor}_{\mathcal{H}}\left(K^{\prime}, K\right)\right|=\operatorname{End}(K)$ and the proof is complete. $\square$

Corollary 13. $K$ is rigid if, and only if, $\mathcal{L} K$ is rigid.

Proof of Corollary 13. If $K$ is rigid, then $\operatorname{End}(K)=$ $1=\operatorname{End}(\mathcal{L} K)$ which implies that $\mathcal{L} K$ is also rigid. If $\mathcal{L} K$ is rigid, then $\operatorname{End}(\mathcal{L} K)=1=\operatorname{End}(K)$ which implies that $K$ is also rigid. $\square$

\section{Some Applications}

\subsection{Dicycles}

It is clear that the dicycles (directed cycles) $\vec{C}_{n}$ on $n$ nodes are objects in $\mathcal{H}$ since they are 1-regular, i.e. for every node $v$ in $\vec{C}_{n}$ there is exactly one arc $x$ and exactly one arc $y$ such that $\partial_{1} x=v=\partial_{0} y$. Dicycles are useful here because they provide trivial validations for aspects of the main results. In particular, since it is well known that $\mathcal{L} \vec{C}_{n}=\vec{C}_{n}$, then substituting this identity into and setting $K=\vec{C}_{n}$ in Corollary 11 and Theorem 12 yields the tautologies

$$
\sum_{\theta \in \Pi_{\vec{C}_{n}}} \operatorname{inj}\left(\vec{C}_{n} / \theta, \vec{C}_{n}\right)=\sum_{\varphi \in \Pi_{\vec{C}_{n}}} \operatorname{inj}\left(\vec{C}_{n} / \varphi, \vec{C}_{n}\right)
$$

and $\operatorname{End}\left(\vec{C}_{n}\right)=\operatorname{End}\left(\vec{C}_{n}\right)$.

\section{2 de Bruijn Digraphs}

Let $\quad S=\{0,1,2, \cdots, d-1\} \quad$ and $S^{n}=\left\{s_{1} s_{2} \cdots s_{n-1} s_{n}: s_{i} \in S\right\}$ be the set of all strings on $S$ of length $n$ and recall that the de Bruijn digraph $B(d, n)$ of dimension $n$ on $d$ symbols has $S^{n}$ as its node set with $x$ an arc in $B(d, n)$ if and only if $\partial_{1} x=s_{2} \cdots s_{n-1} s_{n} s_{n+1}$ when $\partial_{0} x=s_{1} s_{2} \cdots s_{n-1} s_{n}$. Because - as is well known - $B(d, n)$ is $d$-regular, de Bruijn digraphs are objects in $\mathcal{H}$. It is also well known that $B(d, n)$ is the line digraph of $B(d, n-1)$, provided that both digraphs have the same symbol set $S$. These properties lead to the following results for de Bruijn digraphs:

Theorem 14. For any digraph $D$ in $\mathcal{H}$, $\left|\operatorname{Mor}_{\mathcal{H}}(\mathcal{R} D, B(d, n-1))\right|=\left|\operatorname{Mor}_{\mathcal{H}}(D, B(d, n))\right|$.

Proof of Theorem 14. Let $K=B(d, n-1)$ and use the fact that $\mathcal{L} K=\mathcal{L} B(d, n-1)=B(d, n)$ in Theorem 9 to obtain the result. $\square$

Theorem 15. For any de Bruijn digraphs $B(d, n-1)$ and $B(d, n)$,

$$
\begin{aligned}
& \sum_{\theta \in \Pi_{B(d, n-1)}} \operatorname{inj}(B(d, n-1) / \theta, B(d, n-1)) \\
& =\sum_{\varphi \in \Pi_{B(d, n)}} \operatorname{inj}(B(d, n) / \varphi, B(d, n)),
\end{aligned}
$$

where $\Pi_{B(d, n-1)}$ and $\Pi_{B(d, n)}$ are the sets of all partitions of the $B(d, n-1)$ and $B(d, n)$ node sets, respectively.

Proof of Theorem 15. Substitute $K=B(d, n-1)$ and the fact that $\mathcal{L} K=\mathcal{L} B(d, n-1)=B(d, n)$ in Corollary 11.

Theorem 16. $\operatorname{End}(B(d, n-1))=\operatorname{End}(B(d, n))$.

Proof of Theorem 16. Substitute $K=B(d, n-1)$ and $\mathcal{L} K=\mathcal{L} B(d, n-1)=B(d, n)$ in Theorem 12.

\subsection{Kautz Digraphs}

Similar to de Bruijn digraphs, a Kautz digraph $K(d, n)$ of dimension $n$ on $d$ symbols is a $d$-regular digraph that has all strings on $S$ of length $n$ with distinct consecutive symbols, i.e. $s_{i} \neq s_{i+1}$, as its node set and with $x$ an arc in $K(d, n)$ if and only if $\partial_{1} x=s_{2} \cdots s_{n-1} s_{n} s_{n+1}$ when $\partial_{0} x=s_{1} s_{2} \cdots s_{n-1} s_{n}$. Thus, the Kautz digraphs are induced subdigraphs of 
the de Bruijn subdigraphs and since they are $d$ regular, they too are objects in $\mathcal{H}$. It is also well known that for a fixed symbol set $S, K(d, n)$ is the line digraph of $K(d, n-1)$. Since the proofs of the following theorems for Kautz digraphs closely follow those for de Bruijn digraphs, they are stated without proof.

Theorem 17. For any digraph $D$ in $\mathcal{H}$, $\left|\operatorname{Mor}_{\mathcal{H}}(\mathcal{R} D, K(d, n-1))\right|=\left|\operatorname{Mor}_{\mathcal{H}}(D, K(d, n))\right|$.

Theorem 18. For any Kautz digraphs $K(d, n-1)$ and $K(d, n)$,

$$
\begin{aligned}
& \sum_{\theta \in \Pi_{K(d, n-1)}} i n j(K(d, n-1) / \theta, K(d, n-1)) \\
= & \sum_{\varphi \in \Pi_{K(d, n)}} i n j(K(d, n) / \varphi, K(d, n)),
\end{aligned}
$$

where $\Pi_{K(d, n-1)}$ and $\Pi_{K(d, n)}$ are the sets of all partitions of the $K(d, n-1)$ and $K(d, n)$ node sets, respectively.

Theorem 19. $\operatorname{End}(K(d, n-1))=\operatorname{End}(K(d, n))$.

\section{Closing Remarks}

The results in this paper were obtained via a novel application of a functor adjunction borrowed from theoretical biology. This approach not only illustrates the utility of category theory, but also suggests that category theory might play a role in acquiring in a fairly straightforward manner interesting new general mathematical results from otherwise unexpected disparate areas of science.

In closing, it is noted that - whereas digraphs model system topologies - their associated line digraphs model what might be called their associated "interstitial spaces". Consequently, the main results of this paper might be useful during aspects of engineering design processes. For example, if a system's topology is represented by a digraph in category $\mathcal{H}$, it might be important to know from Theorem 12 that a system and its "interstitial space" can be homomorphically "collapsed" onto themselves the same the number of ways.

Acknowledgments: This research was funded by a grant from the Naval Surface Warfare Center
Dahlgren Division's In-house Laboratory Independent Research Program.

\section{References}

1. Döring, A., Isham, C. J. A topos foundation for theories of physics: I. Formal languages for physics. Journal of Mathematical Physics, 2008, vol. 49, 053515, DOI:10.1063/1.2883740.

2. Döring, A., Isham, C. J. A topos foundation for theories of physics: II. Daseinisation and the liberation of quantum theory. Journal of Mathematical Physics, 2008, vol. 49, 053516, DOI:10.1063/1.2883742.

3. Döring, A., Isham, C. J. A topos foundation for theories of physics: III. The representation of physical theories with arrows $\breve{\delta}^{O}(A): \Sigma \rightarrow \mathbb{R}^{z}$. Journal of Mathematical Physics, 2008, vol. 49, 053517, DOI:10.1063/1.2883777.

4. Döring, A., Isham, C. A topos foundation for theories of physics: IV. Categories of systems. Journal of Mathematical Physics, 2008, vol. 49, 053518, DOI:10.1063/1.2883836.

5. Wiels, V., Easterbrook, S. Management of evolving specifications using category theory. In: Proceedings of the $13^{\text {th }}$ IEEE International Conference on Automated Systems Engineering. 1998, p. 12-21.

6. Gebreyohannes, S., Edmonson, W., Esterline, A. Formalization of the Responsive and Formal Design Process using Category Theory. In: Systems Conference (SysCon), 2018 Annual IEEE International, IEEE. 2018, p. 1-8.

7. Kokar, M., Tomasik, J., Weyman, J. Data vs. decision fusion in the category theory framework. FUSION 2001, 2001.

8. Barr, M., Wells, C. Category Theory for Computing Science. Prentice Hall, New York, 2002.

9. Pavlovic, D. Tracing the man in the middle of monoidal categories. arXiv:1203.6324 [cs:LO], 2012.

10. Andrian, J., Kamhoua, C., Kiat, K., Njilla, L. Cyber Threat Information Sharing: A Category-Theoretic Approach. In: Third International Conference on Mobile and Secure Services (MobiSecServ), IEEE. 2017, p. 1-5.

11. Mabrok, M., Ryan, M. Category Theory as a Formal Mathematical Foundation for Model-Based Systems Engineering. Appl. Math. Inf. Sci., 2017, Vol. 11, p. 43-51, DOI: $10.18576 / \mathrm{amis} / 110106$.

12. Wisnesky, R., Breiner, S., Jones, A., Spivak, D., Subrahmanian, E. Using Category Theory to Facilitate Multiple Manufacturing Service Database Integration. J. Comput. Inf. Sci. Eng., 2017, Vol. 17, 021011, DOI: 10.1115/1.4034268.

13. Rudskiy, I. arXiv:1702.04627v1 [q-bio.TO], 2017

14. Phillips, S. A General (Category Theory) Principle for General Intelligence: Duality (Adjointness). In: Artificial General Intelligence. AGI 2017. Everitt, T., Goertzel, B., Potapov, A. Eds. Lecture Notes in Computer Science, Vol. 10414; Springer, Cham, DOI: 10.1007/978-3-319-63703-7 6.

15. Haruna, T., Gunji, Y-P. Duality between decomposition and gluing: A theoretical biology via adjoint functors. Biosystems, 2007, vol. 90, 716-727.

16. Haruna, T., Gunji, Y-P. An Algebraic Description of Development of Hierarchy. International Journal of Computing Anticipatory Systems, 2008, vol. 20, 131-143.

17. Zerbina, D., Birney, E. Velvet: Algorithms for de novo short read assembly using de Bruijn graphs. Genome Research, published in advance in March 2008, DOI: 10.1101/gr.074492.107.

18. Tvrdik, P., Harbane, R., Heydemann, M-C. Uniform homomorphisms of de Bruijn and Kautz networks. Discrete Applied Mathematics, 1998, vol. 83, 279-301.

19. Hell, P., Nešetřil, J. Graphs and Homomorphisms. Oxford University Press Inc., New York, 2004. 